\title{
Investigation of rheological and thermal properties of recycled poly (ethylene terephthalate)/ hyperbranched polyester composites
}

\begin{abstract}
To investigate the effect of hyperbranched polyester (HBPET) and concentration on the rheology and thermal properties of recycled polyethylene terephthalate (RPET). Different amounts of hyperbranched polyesters (HBPET), $3 \mathrm{wt} \%$ and $5 \mathrm{wt}$ $\%$ were added to the RPET. The influence of HBPET on the rheological and thermal properties of the RPET was investigated. Thermal stability and rheological behavior of the pure recycled PET (RPET- 0 ) and RPET- 3 and RPET- 5 composites prepared by melt compounding were tested by Thermogravimetric analysis (TGA), Differential Scanning Calorimetry (DSC) and parallel-plate rheometer. RPET-3 and RPET-5 composites were in a good agreement with improved rheological characteristics and led to significant enhancement in thermal stability. Decrease in the complex viscosity $(\eta)$ of the blends was observed with increasing content of HBPET
\end{abstract}

Keywords: complex viscosity, recycled PET, hyperbranched polyester, thermal properties
Volume 4 Issue 6 - 2018

\author{
Haroon AM Saeed, ${ }^{1,2}$ Yassir A Eltahir, ${ }^{1,2}$ Yumin \\ Xia,' Wang Yimin' \\ 'College of Materials Science and Engineering, State Key \\ Laboratory for Modification of Chemical Fibers and Polymer \\ Materials, Donghua University, China \\ ${ }^{2}$ Faculty of Textiles, University of Gezira, Sudan
}

\begin{abstract}
Correspondence: Haroon AM Saeed, PhD, Assistant Professor of Material Science \& Engineering, Faculty of Industries Engineering \&Technology, University of Gezira, Po. Box: 20, Sudan, Tel +249| I2322234, Email haronsaeed75@gmail.com
\end{abstract}

Received: February 15, 2018 | Published: November 19, 2018

\section{Introduction}

During the last two decades, hyperbranched polymers have become the focus of interdisciplinary research, and received considerable attention due to their unique chemical and physical properties as well as their potential applications. ${ }^{1-5}$ Hyperbranched polymers (HBPs) have been discovered at the end of 1980s. After the linear, branched, and crosslinking polymers, hyperbranched polymers become the fourth generation macromolecular architecture. ${ }^{1,6}$

Since rheological plays key roles in the properties of the resulting products, more sophisticated control of these factors may facilitate even wider technological applications. One of methodologies used to control rheological of recycled polyesters is to incorporate branched structures via small amount of mult-functional monomer. However, the use of mult-functional branching reagents always results in a cross-linked network. Recently, it was demonstrated that using an end-capped reagent prevented cross-linking, and thus facilitated the preparation of polyesters with a higher level of branching hyperbranched polymers have emerged as popular alternatives to dendrimers in the last 15 years. ${ }^{7,8}$

The properties and applications of conventional polymers can be improved by blending with other polymers to create new materials with tailored properties and improved performance. Thus, there has been considerable interest in recent years in exploring the potential of different polymer blends, particularly in view of the opportunities to combine the useful properties of each polymer while minimizing their undesired characteristics. ${ }^{9}$

Poly(ethylene terephthalate) (PET), as a high-strength and highmodulus thermoplastic polyester, is widely employed in many applications such as synthetic fibers films and beverage, food, other liquid containers and parts in automotives and electronics due to its superior thermal and mechanical properties, low permeability, and chemical resistance. ${ }^{9-11}$ Extensive use of PET in the bottling industry makes postconsumer PET bottles a readily available source for the PET recycling industry. However, during the recycling processes PET undergoes chemical, mechanical, thermal and oxidative degradation. Degradation of RPET renders it unsuitable for many applications.

Polymer rheological properties help to formulate a polymer system in respect to its processing characteristics, to optimizing the processing conditions such as extrusion, injection molding and melt spinning, which is highly dependent on temperature, molecular weight and shear rate. It is also give an insight into the physical properties of the system because there is interplay between the processing conditions, structures, and properties. ${ }^{12}$

hyperbranched polyester (HBPET) possess a compact, highly branched, three-dimensional (3D) structure, which has a high density of functional end groups, less of entanglements and inherently low melt and solution viscosity. ${ }^{13}$ The combination of these properties, low viscosity, less entanglement and high reactivity, makes HBPET attractive candidates for reactive polymer blending. The HBPET additives are able to behave as lubricants during processing and as self-compatibilizing toughening agents in the final blend formulation. TJ Mulkern et al. ${ }^{14}$ studied polystyrene/ hyperbranched polyester blends; they found that a significant drop in the blend viscosity occurs immediately on addition of HBP. O Monticelli et al. ${ }^{15}$ and $\mathrm{Z}$ Fan et al. ${ }^{16}$ studied the blends of polyamide and hyperbranched polymers, they found that the hyperbranched polymers strongly modified the rheological behavior of polyamide. Moreover, X Li et al. ${ }^{17}$ Study of blends of linear poly(ether ether ketone) of high melt viscosity and hyperbranched poly(ether ether ketone), the rheological measurements show that blending LPEEK with as little as $1 \mathrm{wt} \%$ HPEEK results in about $17 \%$ reduction of melt viscosity. 
Up to now, a few papers, which are mentioned above have dealt with hyperbranched polymers as modifiers, but no mention for recycled PET modified by HBPET (which is interesting particularly from the environmental point of view) was found in available literature.

In this article, HBPET was used as rheological modifier for recycled PET, and melt rheological properties and thermal stability of the pure recycled PET (RPET-0) and RPET- 3 and RPET-5 composites were investigated. The description of the synthesized HBPET ${ }^{18}$ and it is application as nucleation agent for recycled PET $^{19}$ was published by the authors elsewhere. Complex viscosity ( $\eta)$, storage modules (G') and loss modulus (G') at different range of frequency has been discussed. Thermal behavior which includes thermal stability and thermal properties of the blends were also investigated.

\section{Experimental}

\section{Materials}

Adipic acid, phloroglucinol, and p-Toluene sulfonic acid (p-TSA) were purchased from Sinopharm Chemical Reagent Co. Ltd (China). Recycled PET (RPET-0) with an intrinsic viscosity of $0.85 \mathrm{dL} / \mathrm{g}$ (measured in a $60: 40 \mathrm{wt} \%$ solvent of phenol and 1,1,2,2-tetrachloroethane at $25^{\circ} \mathrm{C}$ ), MFI of $44 \mathrm{~g} / 10 \mathrm{~min}$ at $270^{\circ} \mathrm{C}$ with a weight of $2.16 \mathrm{~kg}$, acetone and hexane were obtained through Donghua university chemical store. All reagents and solvents were commercial grade and used as received.

\section{Synthesis of HBPET}

The HBPET was synthesized with the divergent approach in a single step melt polycondensation reaction of adipic acid $(27 \mathrm{~g}, 0.18 \mathrm{~mol})$ and phloroglucinol $(15 \mathrm{~g}, 0.12 \mathrm{~mol})$ as a core, the molar ratio of adipic acid and phloroglucinol was 3:1. The phloroglucinol was charged with adipic acid kept in a $250 \mathrm{ml}$ four-necked, round-bottom flask placed over an oil bath and equipped with a thermometer, mechanical stirrer, nitrogen inlet, and Dean-Stark apparatus. The reaction mixture was slowly heated to $170^{\circ} \mathrm{C}$. After the reactants were completely melted, the temperature was maintained at $160^{\circ} \mathrm{C}$ with a continuous nitrogen flow for $9 \mathrm{~h}$. p-Toluene sulfonic acid (p-TSA) $(0.05$ weight $\%$ on the basis of the weight of adipic acid) was used as the catalyst for the esterification. The crude products were collected as pale brown color, and were purified and precipitated from acetone in hexane to remove the un-reacted materials and catalyst. Then the products were dried at $60^{\circ} \mathrm{C}$ under vacuum. The HBPET weight average molecular weight $\left(\mathrm{M}_{\mathrm{w}}\right)$ was $9214 \mathrm{~g} / \mathrm{mol}$ calculated from (GPC). Thermogravimetric analysis of the HBPET showed a maximum mass loss rate at about $500^{\circ} \mathrm{C}$. The degree of branching (DB) of the HBPET was 0.48 . The possible structure of the synthesized HBPET was shown in scheme (1).

\section{Blends preparation}

Recycled poly (ethylene terephthalate) (RPET) was dried in a vacuum oven at $120^{\circ} \mathrm{C}$ for $24 \mathrm{~h}$, and the HBPET was dried in an oven at $80^{\circ} \mathrm{C}$ for $24 \mathrm{~h}$ before preparing the blends to remove moisture in order to minimize the possibility of hydrolysis. RPET and HBPET were mixed together with $3 \%$ and $5 \%$ wt of HBPET, and then melts-blended in (a SHJ-20 type self-wiping, co-rotating twin-screw extruder with six heating sections, made by Nanjing Giant Machinery Co. (China)), operating at a screw speed of 100rpm, and with temperatures of 250 , $255,260,260,260,250$, and $180^{\circ} \mathrm{C}$ from the first section to the die. The molten extrudates were quenched by cold water at once and then cut into pellets.

\section{Melt rheological measurements}

The rheological measurements were performed according to ASTM Standard D 4440-95, using a HAAKE MARS, Modular Advanced Rheometer system (Germany) with parallel-plate rheometer in oscillation mode under a nitrogen atmosphere at $280^{\circ} \mathrm{C}$. The plate diameter was $25 \mathrm{~mm}$, and the gap ranged from 1.2 to $1.95 \mathrm{~mm}$. A frequency range between 0.1 and $100 \mathrm{rad} / \mathrm{s}$ and a strain within the linear viscoelastic range were used.

\section{Thermogravimetric Analysis (TGA)}

Thermal stability of pure recycled PET (RPET-0) and RPET-3 and RPET- 5 composites performed using theromgravimetric analysis (TGA) on a simultaneous thermal analyzer (Netzsch TG209F1). The sample weighed between $5-10 \mathrm{mg}$. The scans were run from room temperature to $600^{\circ} \mathrm{C}$ at rate of $20^{\circ} \mathrm{C} / \mathrm{min}$ under nitrogen flow.

\section{Differential Scanning Calorimetry (DSC)}

Thermal characterization of pure recycled PET (RPET-0) and RPET-3 and RPET-5 composites was performed with DSC Q20 (TA Instrument-waters LLC). Sealed aluminium pans containing 5-10mg of the samples were used in all the experiments. To eliminate the thermal history, all the samples were heated up to $180^{\circ} \mathrm{C}$, then rapidly cooled to $-50^{\circ} \mathrm{C}$. The actual measurements reported were performed during a second heating cycle from $50-600^{\circ} \mathrm{C}$ at a heating rate of $10^{\circ} \mathrm{C} / \mathrm{min}$

\section{Results and discussion}

\section{Rheological measurements}

Rheological measurements are very important for quality control of raw materials, optimization of manufacturing processes and forecasting the performance of a material. ${ }^{20}$ The hyperbranched polymers as a subgroup of dendritic polymers are very well known for their reducing effect on viscosity. It is also mentioned in the literature that the smaller size of the dendritic domains causes a further reduction in viscosity. ${ }^{21}$

The influence of the addition of HBPET-A on the rheological behavior of recycled PET was also of high interest. A lowering of the melt viscosity and thus, an improvement of the processing properties is expected. The complex viscosity $\left(\eta^{*}\right)$ of the pure recycled PET (RPET-0) and RPET-3 and RPET- 5 composites containing $3 \mathrm{wt} \%$ and $5 \mathrm{wt} \%$ of HBPET-A are shown as functions of frequency in Figure 1. The Flow curve for complex viscosity ( $\left.\eta^{*}\right)$ of RPET- 0 and RPET- 3 and RPET-5 composites display a typical property of pseudo-plastics nonNewtonian or shear-thinning behavior. Moreover, the flow curves for show that the complex viscosity decreased with increasing shear rate. The modification of recycled PET with HBPET-A leads to an obvious reduction of the complex viscosity. Even a small concentration of HBPET-A already results in a significant decrease of the complex viscosity. 


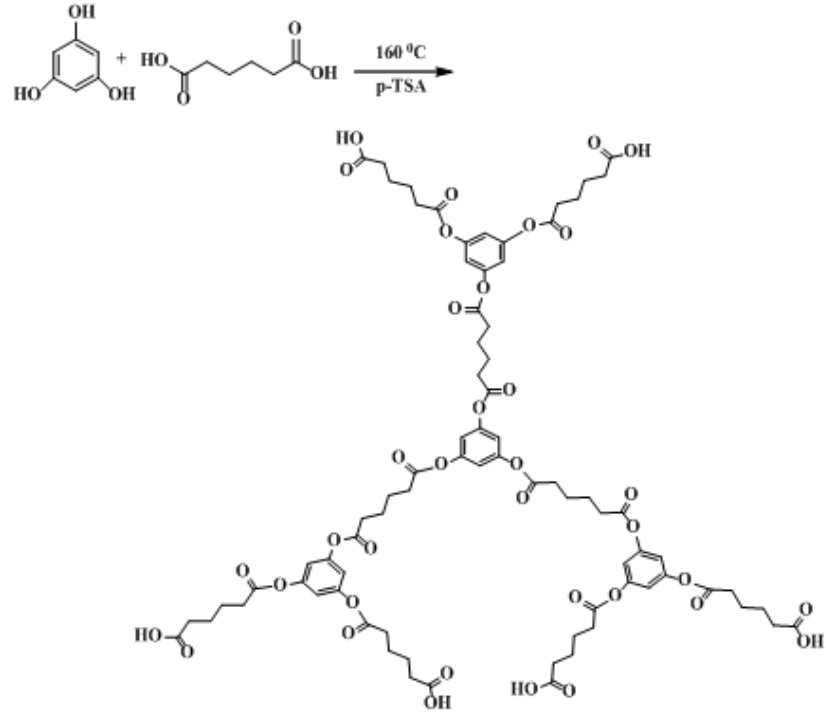

Scheme I Synthesis route and possible structure of HBPET.

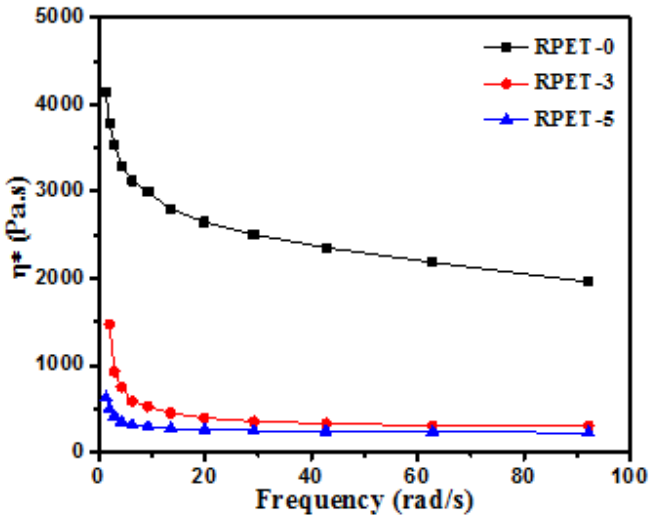

Figure I Complex viscosity of RPET- 0 and RPET-3 and RPET- 5 composites vs. frequency $\left(T=280^{\circ} \mathrm{C}\right)$.

After adding $3 \mathrm{wt} \%$ of HBPET-A (RPET-3), the zero-shear viscosity (viscosity extrapolated to a frequency of $0 \mathrm{rad} / \mathrm{s}$ ) of recycled PET was changed from 4142 (Pa. s) (0 wt $\%$ of HBPET-A) to 1471 (Pa.s) (3 wt \% of HBPET-A). With increasing the amount of HBPET-A in the composite a continuous reduction of the complex viscosity was observed at a concentration of $5 \mathrm{wt} \%$ it was reached to 626 (Pa.s) ( $5 \mathrm{wt} \%$ of HBPET-A). This behavior has been reported previous publications. ${ }^{14,17,22}$ The decrease of composites complex viscosity is due to the good dispersion interaction between recycled PET and HBPET-A.

Complex viscosity $(\eta)$ can be decomposed into the storage modulus $\left(G^{\prime}\right)$ and loss modulus $\left(G^{\prime \prime}\right)$. The elastic part of $(\eta)$ is described by the $\mathrm{G}^{\prime}$ that shows the size of the recoverable energy stored in melting sample, while the viscous part is described by the $\mathrm{G}^{\prime \prime}$ that shows the size of the non recoverable energy. However, the effect of HBPET content on storage modulus $\left(\mathrm{G}^{\prime}\right)$ and loss modulus $\left(\mathrm{G}^{\prime \prime}\right)$ of the RPET3 and RPET- 5 composites can be observed in Figure 2 and Figure 3 respectively. Both storage modulus $\left(\mathrm{G}^{\prime}\right)$ and loss modulus $\left(\mathrm{G}^{\prime \prime}\right)$ increased significantly with HBPET concentration at whole frequency range 0.1-100 rad/s. With increasing HBPET loading, the dependence of storage modulus and loss modulus on frequency increases. It is believed that the degree of dependence of storage modulus $\left(\mathrm{G}^{\prime}\right)$ and loss modulus $\left(\mathrm{G}^{\prime \prime}\right)$ on the frequency reflects sensitively the effect of HBPET on the viscoelastic properties of the blends. This pseudosolid-like behavior is attributed to the formation of a space-filling interconnected network of HBPET particles. This percolated threedimensional network acts like a weak solid and causes significant enhancement of the storage modulus, as well as the loss modulus $\left(\mathrm{G}^{\prime \prime}\right)$.

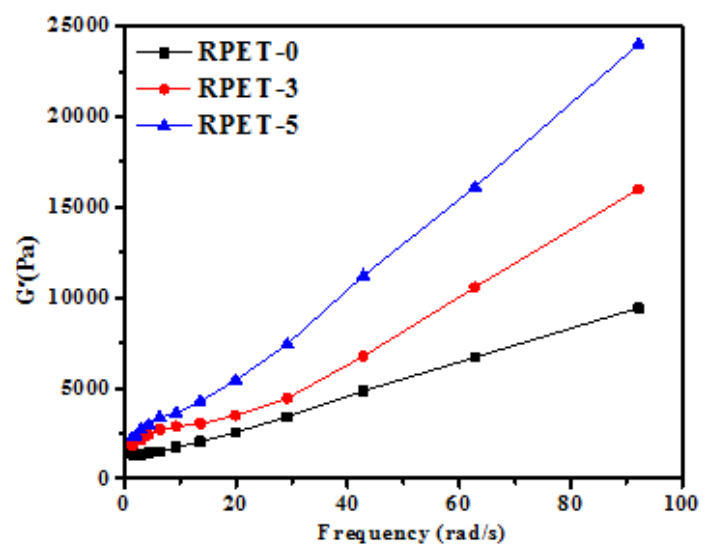

Figure 2 Storage modulus (G') vs. frequency of RPET-0 and RPET-3 and RPET5 composites $\left(\mathrm{T}=280^{\circ} \mathrm{C}\right)$.

The storage modulus $\left(\mathrm{G}^{\prime}\right)$ as a function of loss modulus $\left(\mathrm{G}^{\prime \prime}\right)$ was plotted for the RPET-0 and RPET- 3 and RPET-5 composites Figure 4 Compared with RPET-0, all composite samples show rubber-like behavior which is indicated as $G^{\prime}$ secondary plateau in the range of low frequencies. This phenomenon is especially distinct in Figure 4, where the viscoelastic liquid of PET- $0\left(\mathrm{G}^{\prime \prime}>\mathrm{G}^{\prime}\right)$. These properties are attributed to physical network structures in the composites. 
Plotting the storage modulus (G') and the loss modulus (G') against the frequency was shown in Figure 5. From this figure it clearly that, the curves of the loss modulus for all samples are located above of that of the storage modulus. It can be seen that, the viscous part of the complex melt viscosity is represented by the loss modulus (G'), whereas the elastic part is described by storage modulus (G"). However, these composites show mainly a viscous behavior at $280^{\circ} \mathrm{C}$ more than an elastic behavior. This agrees with the observation that the RPET- 0 and RPET- 3 and RPET-5 composites were extremely fluid when they were taken out of the extruder. ${ }^{13}$

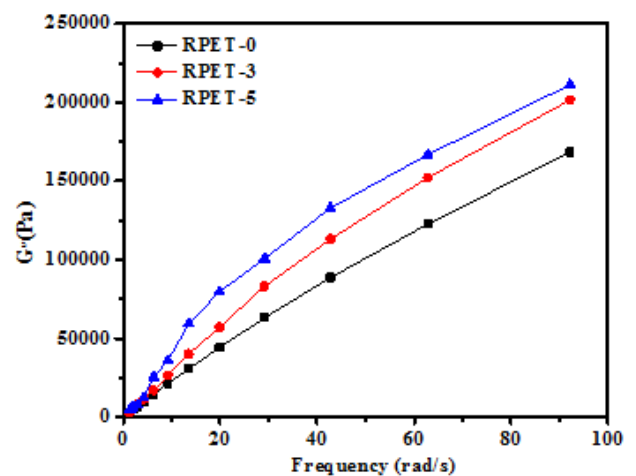

Figure 3 Loss modulus vs. frequency of the RPET- 0 and RPET- 3 and RPET-5 composites vs. frequency $\left(T=280^{\circ} \mathrm{C}\right)$.

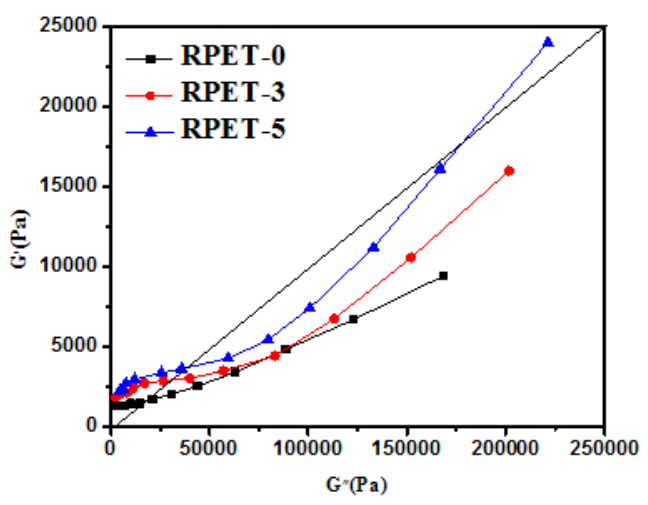

Figure 4 Storage modulus $\left(\mathrm{G}^{\prime}\right)$ as a function of loss modulus $\left(\mathrm{G}^{\prime \prime}\right)$ at $\left(280^{\circ} \mathrm{C}\right)$.

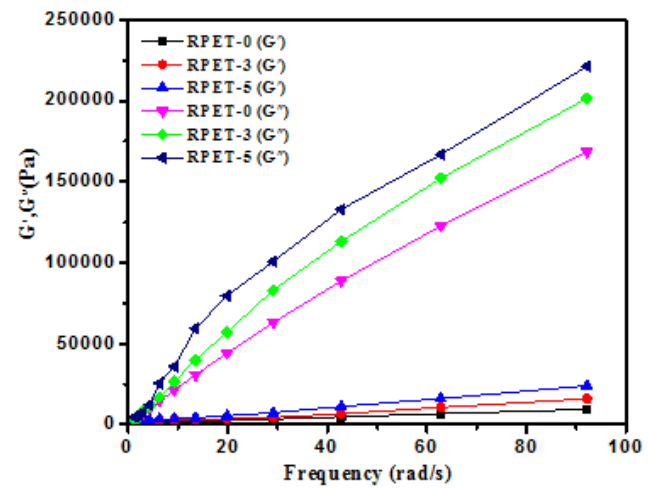

Figure 5 Plotting the storage modulus ( $\left.G^{\prime}\right)$ and the loss modulus (G') vs frequency at $\left(280^{\circ} \mathrm{C}\right)$.

\section{Thermal properties}

The thermal stability of a polymer can provide an important guidance for its processing and application. The studies of thermal behavior, not only can explain the behavior of a polymer at high temperature, but also provide assistance for the establishment of selection criteria for specific applications of polymer materials. ${ }^{23}$ The corresponding initial decomposition temperatures $\left(\mathrm{T}_{5}\right)$ at which the weight loss of the sample reaches $5 \mathrm{wt} . \%$, and the temperatures for $10 \%$ gravimetric loss $\left(\mathrm{T}_{10}\right)$, which is an important evidence for thermal stability and char yield at $600^{\circ} \mathrm{C}$ are summarized in Table 1.

Figure 6 reports the trends of weight loss (\%) vs temperature resulting from the thermogravimetric analysis. It can be seen that the thermogravimetric temperature of the RPET-3 and RPET-5 composites shifts to higher temperature than that of RPET- 0 . This fact indicates that there is a strong interaction between the HBPET and RPET. Similar results of high thermogravimetric temperature of PET/Silica nanocomposites. ${ }^{24,25}$ Moreover, it can be found that there is no significant difference in char residue of the RPET-3 and RPET5 composites than that of RPET- 0 . These additional weights may be of un-decomposed of HBPET within this range of the temperature. However, it can be concluding that HBPET could enhance the thermal stability of the composites.

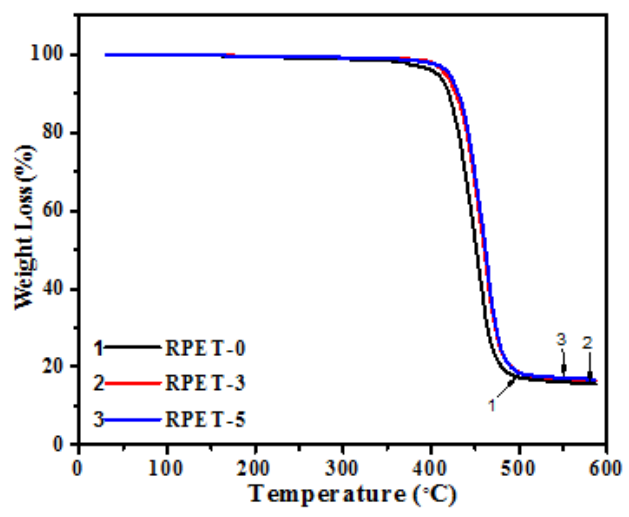

Figure 6 Thermogravimetric curves for RPET-0 and RPET-3 and RPET-5 composites.

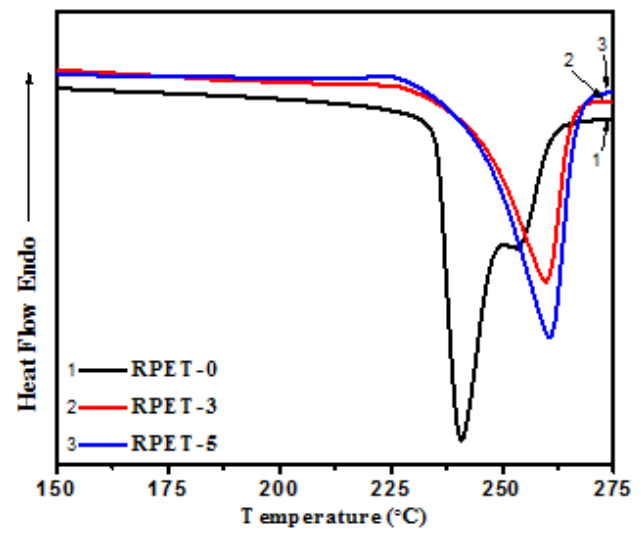

Figure 7 DSC curves of heating measurements of RPET-0 and RPET- 3 and RPET-5 composites. 
Table I Thermal properties data

\begin{tabular}{lllllll}
\hline Sample & $\mathbf{T c}\left({ }^{\circ} \mathbf{C}\right)$ & $\mathbf{T m}\left({ }^{\circ} \mathbf{C}\right)$ & $\Delta \mathbf{H m}(\mathbf{J} / \mathbf{g})$ & $\mathbf{T 5 \%}\left({ }^{\circ} \mathbf{C}\right)$ & $\mathbf{T 1 0 \%}\left({ }^{\circ} \mathbf{C}\right)$ & Char.Res. $(\%)$ \\
\hline RPET-0 & 175 & 240 & 30 & 405 & 418 & 15 \\
RPET-3 & 214 & 259 & 48 & 414 & 426 & 16 \\
RPET-5 & 217 & 260 & 49 & 420 & 430 & 16 \\
\hline
\end{tabular}

The DSC curves of heating and cooling measurements of the RPET- 0 and RPET-3 and RPET-5 composites are shown in Figure 7 and Figure 8, respectively. The data of thermal properties listed in Table1 are calculated from Figure 7 and Figure 8, it can be seen that the presence of HPET in RPET- 3 and RPET- 5 composites increased the crystallizing temperature by $39^{\circ} \mathrm{C}$ for RPET-3 and $41^{\circ} \mathrm{C}$ for RPET-5 composites when compared with RPET-0. Moreover, the melting points RPET- 3 and RPET-5 composites are higher than that of RPET- 0 by $20^{\circ} \mathrm{C}$, these results in agreement with the previous publications. ${ }^{24,25}$ It is thought that the increase in $\mathrm{T}_{\mathrm{m}}$ may result from the well dispersed HBPET that make the RPET segment form more perfectible crystals during the crystallization. However, these results indicated that the RPET modified with HBPET could be regarded as a network connection point due to the good compatibility of the HBPET and the reaction between RPET and HBPET.

\section{Conclusion}

The rheological behavior and thermal properties of recycled PET and of it is composites with $\mathrm{OH}$-terminated hyperbranched polyester (HBPET) were investigated. It was found that the viscosity of recycled PET, initially Newtonian, becomes pseudo-plastic when the HBPET is added; the greater the content of the HBPET, the higher its effect on the viscosity of the recycled PET, particularly at lower shear rates. There is evidence that the addition of an HBPET influences the increase in the molecular weight and complex viscosity of recycled PET. Moreover, the addition of HBPET increases the crystallizing temperature and melting point of the RPET. However, HBPET not only acts as a thermal barrier but also as heat storage.

\section{Acknowledgements}

None.

\section{Conflict of interest}

Author declares there is no conflict of interest.

\section{References}

1. Zhang X. Hyperbranched aromatic polyesters: From synthesis to applications. Progress in Organic Coatings. 2010;69(4):295-309.

2. Fan $Z$, Lederer A, Voit B. Synthesis and characterization of $A_{2}+B_{3}$-type hyperbranched aromatic polyesters with phenolic end groups. Polymer. 2009;50(15):3431-3439.

3. Zeiner T, Enders S. Phase behavior of hyperbranched polymer solutions in mixed solvents. Chemical Engineering Science. 2011;66(21):5244-5252.

4. Zeiner T, Browarzik C, Browarzik D, et al. Calculation of the (liquid plus liquid) equilibrium of solutions of hyperbranched polymers with the lattice-cluster theory combined with an association model. J Chemical Thermodynamics. 2011;43(12):1969-1976.
5. Zeiner T, Browarzik D, Enders S. Calculation of the liquid-liquid equilibrium of aqueous solutions of hyperbranched polymers. Fluid Phase Equilibria. 2009;286(2):127-133.

6. Wei Z, Hao X, Kambouris PA, et al. One-pot synthesis of hyperbranched polymers using small molecule and macro RAFT inimers. Polymer. 2012;53(7):1429-1436.

7. Miao H, Bao F, Cheng L, et al. Fluorinated modification of hyperbranched polyesters used for improving the surface property of UV curing coatings. J Fluorine Chemistry. 2010;131(12):1356-1361.

8. Zhang X. Synthesis and characterization of hyperbranched polyesters based on isophthalic acid and trimethylolpropane. J Macromolecular Science Part a-Pure and Applied Chemistry. 2011;48(2):128-134.

9. Massa D, Shriner KA, Turner SR, et al. Novel blends of hyperbranched polyesters and linear polymers. Macromolecules. 1995;28(9):3214-3220.

10. Gokkurt T, Durmus A, Vedat Sariboga, et al. Investigation of thermal, rheological, and physical properties of amorphous poly (ethylene terephthalate)/organoclay nanocomposite films. J Applied Polymer Science. 2013;129(5):2490-2501.

11. Yesil S. Effect of carbon nanotube reinforcement on the properties of the recycled poly (ethylene terephthalate)/poly(ethylene naphthalate) (r-PET/ PEN) blends containing functional elastomers. Materials \& Design. 2013;52:693-705.

12. Li S-C, Lu L-N. Melt rheological properties of reactive compatibilized HDPE/PET blends. J Applied Polymer Science. 2008;108(6):3559-3564.

13. Mingtao R, Wang J, Yao M, et al. Influences of hyperbranched poly(amideester) on the properties of poly(butylene succinate). Materials Chemistry \& Physics. 2013;139(2-3):988-997.

14. Mulkern TJ, Tan NCB. Processing and characterization of reactive polystyrene/hyperbranched polyester blends. Polymer. 2000;41(9):31933203 .

15. Monticelli O, Oliva D, Russo S, et al. On blends of polyamide 6 and a hyperbranched aramid. Macromolecular Materials \& Engineering. 2003;288(4):318-325.

16. Fan Z, Jaehnichen $\mathrm{K}$, Desbois $\mathrm{P}$, et al. Blends of Different Linear Polyamides with Hyperbranched Aromatic $\mathrm{AB}_{2}$ and $\mathrm{A}_{2}+\mathrm{B}_{3}$ Polyesters. $J$ Polymer Science Part a-Polymer Chemistry. 2009;47(14):3558-3572.

17. Li X, Zhang S, Wang H, et al. Study of blends of linear poly (ether ether ketone) of high melt viscosity and hyper branched poly (ether ether ketone). Polymer International. 2011;60(4):607-612.

18. Saeed HAM, Yimin W. Synthesis and characterization of $\mathrm{A}_{2}+\mathrm{B}_{3}$ type hyper branched aromatic-aliphatic polyester with carboxyl end groups. Russian J Applied Chemistry. 2013;86(10):1569-1575.

19. Saeed HM, Eltahir YA, Xia Y, et al. Non-isothermal crystallization kinetics and nucleation activity of hyperbranched polyester (HBPET) in recycled PET. Polymer Bulletin. 2014;71(3):595-612. 
20. Mehmood U, et al. Rheological and Spectroscopic Characterization of Recycled Blend Containing Commonly Used Plastics of Daily Life. $J$ Materials Science and Engineering A: Structural Materials: Properties, Microstructure and Processing. 2012;2(7):494-500.

21. Ganjaee Sari M, Stribeck N, Moradian S, et al. Dynamic mechanical behavior and nanostructure morphology of hyper branched-modified polypropylene blends. Polymer International. 2014;63(2):195-205.

22. Han K, Li W, Wu C, et al. Study on hyper branched polyesters as rheological modifier for Spandex spinning solution. Polymer International. 2006;55(8):898-903.
23. Dolbin IV, Burya AI, Kozlov GV. The structure and thermal stability of polymer materials: A fractal model. High Temperature. 2007;45(3):313316.

24. Liu W, Tian X, Cui P, et al. Preparation and characterization of PET/silica nanocomposites. J Applied Polymer Science. 2004;91(2):1229-1232.

25. Gu XH, Zeng P, Zhou JL, et al. Preparation and characterization of poly (ethylene terephthalate) incorporated with secondary-modified montmorillonite. Iranian Polymer J. 2014;23(4):249-255. 